Anuario de Estudios Medievales

43/1, enero-junio de 2013, pp. 259-289

ISSN 0066-5061

doi:10.3989/aem.2013.43.1.09

\title{
SALUTE DEI RE, SALUTE DEL POPOLO. MANGIARE E CURARSI NELLA SICILIA TARDOMEDIEVALE ${ }^{1}$
}

\author{
THE KING'S HEALTH AND THE PEOPLE'S HEALTH. \\ EATING AND CARE OF THE SELF IN LATE MEDIEVAL SICILY
}

\author{
DANIELA SANTORO \\ Università degli Studi di Palermo
}

\begin{abstract}
Riassunto: Mangiare e curarsi sono due dimensioni fortemente connesse al corpo, tra bisogno e piacere. Due dimensioni dense di sfumature sociali e locali, religiose ed economiche, che implicano conoscenze e terapie diverse a seconda dell'età e del sesso ma soprattutto del ruolo sociale. Con un'attenzione particolare a corti (signorili, vescovili, reali) e città, il lavoro focalizza metodi, usi e pratiche curative e alimentari di sovrani e isolani.
\end{abstract}

Parole chiave: salute; alimentazione; terapia; rimedi; ricreazione; monarchia; popolo; Sicilia medievale.

\begin{abstract}
Dining and personal health care are two dimensions which are strongly connected to the body, between need and pleasure. They are two dimensions which have many social, local, religious and economic implications involving knowledge and different therapies related to age and gender. The social role is equally important. With a focus on the court (noble, episcopal, real) and the cities, the present work focuses on the methods and the uses of healing practices of both kings and islanders.
\end{abstract}

Keywords: health; diet; therapy; remedies; recreation; monarchy; people; Medieval Sicily.

\section{SOMMARIO}

1. Introduzione.- 2. Cibo per la corte.- 3. Cibo per la città.- 4. Ricreazione.-

5. Conclusioni.- 6. Bibliografia citata.

\footnotetext{
${ }^{1}$ Abbreviazioni utilizzate: ACA = Archivo de la Corona de Aragón; ACFUP = Acta Curie Felicis urbis Panormi (Collana di Atti della città di Palermo); $\mathrm{ACP}=$ Archivio Comunale di Palermo; ASP = Archivio di Stato di Palermo; TRP = Tribunale del Real Patrimonio . Ringrazio Laura Sciascia per i preziosi suggerimenti.
} 


\section{INTRODUZIONE}

Alla parola "cibo" gli ospiti, con le loro vecchie teste chine sulle mani giunte, ricordarono che s'erano giurati

di non pronunciar parola su quell'argomento, e in cuor loro rafforzarono il voto: non gli avrebbero neppure dedicato un pensiero! Karen Blixen, Il pranzo di Babette

In uno sfavillante Palazzo reale addobbato con drappi lucenti e tappeti colorati, nel giorno della sua incoronazione a re di Sicilia, Ruggero II d'Altavilla aveva offerto un banchetto opulento servito in piatti e bicchieri d'oro e d'argento: nel superbo scenario palermitano in cui anche coloro che servivano a tavola vestivano di seta ${ }^{2}$, quel pasto dal "valore simbolico notevole", era occasione per esibire benessere e prestigio ${ }^{3}$. Un contesto diverso quello profilato nel privilegio con il quale Guglielmo II, nel 1182, confermava alla chiesa di Monreale la bolla d'oro di fondazione: al sovrano erano dovuti duos panes et tantum de $v$ vino $^{4}$, semplice e sobrio pasto dai richiami più liturgici e rituali che economici e alimentari ${ }^{5}$. Due situazioni differenti alla cui messa a fuoco sono utili le considerazioni di Roland Barthes sulla nutrizione che non è mai, solamente, une collectione de produits, justiciables d'études statistiques ou diététiques. C'est aussi et en même temps un système de communication, un corps d'images, un protocole d'usages, de situations et de conduites ${ }^{6}$. Ogni alimento viene investito di un valore esteriore e apparente, molto simile a quello attribuito agli abiti, utilizzato per trasmettere le differenze sociali e codici precisi, generalmente condivisi, sono utilizzati a definire la natura del cibo, nobile o mediocre ${ }^{7}$.

Una tensione avvolge dunque il cibo, con estremi e significati variabili a seconda delle aree e delle età: mangiare è segno di potenza, mangiare libera gli istinti, è occasione di cedimento ai sensi ${ }^{8}$ e fa male in un' ottica, quale quella dei manuali sulla salute, che mira alla temperanza e al mantenimento

${ }^{2}$ Alessandro di Telese, De rebus gestis, 1. II, capp. 5 e 6, p. 103; S. Tramontana, L'effimero nella Sicilia normanna, p. 29.

${ }^{3}$ S. Tramontana, Giochi, feste, spettacoli, p. 323. Mangiare "e dar da mangiare" sono "parte del funzionamento del potere" e la tavola "offre lo spettacolo della potenza dell'ospite", J.-C. Schmitt, Il gesto, p. 205.

${ }^{4}$ I documenti inediti, doc. LXXIII, p. 181.

${ }^{5}$ S. Tramontana, Modelli alimentari di Sicilia, p. 123.

${ }^{6}$ R. Barthes, Pour une psycho-sociologie de l'alimentation, p. 979.

${ }^{7}$ A.J. Grieco, Alimentazione e classi sociali, p. 380. Esemplificativa la connessione tra verdure e classi sociali inferiori diventata "relazione quasi simbiotica", ibidem, p. 379.

${ }^{8}$ In una visione complessiva che tende a mortificare la carne, intesa come sessualità, gli alimenti sono accolti (regime disseccante) o rifiutati in base alla loro capacità di incidere sugli umori sessuali, M. Montanari, Alimentazione, pp. 66-67. 
di un equilibrio degli umori adattato al temperamento della persona, sana o malata. Eccesso e sregolatezza da un lato, controllo e astinenza dall'altro: una perenne oscillazione tra Quaresima e Carnevale che condiziona corpi e menti.

Oltre all'abbondanza, necessaria a esibire ricchezza e potere, il cibo a corte prevede una dimensione ludica all'insegna della festa, dello svago che ridà tono e contribuisce ad appagare corpo e spirito. Lento e senza limiti a corte, il tempo della festa va veloce per gli altri. Occasione di scompostezza del corpo e offuscamento della mente, feste e banchetti vengono tenuti a freno da ripetute norme suntuarie: nel 1308 Federico III d'Aragona, in un'operazione complessiva di controllo della società isolana, emanava norme specifiche riguardanti generalia convivia nuptiarum, con la limitazione di un solo giorno nel caso di parenti degli sposi venuti de exteribus partibus ad nuptias ${ }^{9}$. Il regime alimentare del re non si era attenuto a rigidi comportamenti: in viaggio con moglie e figli da Palermo verso Enna dove ama trascorrere i mesi estivi, Federico III muore nel giugno 1337, già da tempo colpito da gotta nelle mani e nei piedi ${ }^{10}$. La quantità prevale sulla qualità, la ricerca del gusto su eventuali limitazioni a tutela della salute e per molti re, non Federico II che segue strettamente i consigli alimentari dei suoi medici, il cibo è causa di malattia.

Per Federico II il medico di corte Teodoro di Antiochia compilava, su apposita richiesta dell'imperatore, un trattato di igiene in forma di lettera, l'Epistola Theodori philosophi ad imperatorem Fridericum in cui il consiglio su cui si insiste è mangiare poco ${ }^{11}$, e poi di utilizzare la frutta ut medicamen$t_{i s^{12}}$, di passeggiare dopo aver pranzato per amena et delectabilia loca e di concedersi del buon vino -al vino si riconoscono, oltre che significati simbolici, importanti virtù igieniche e medicinali: facilita la digestione, è adatto a tutte le età, primo rimedio alla debolezza dell'organismo malato ${ }^{13}-$ quod non erit acetosum, turbidum, novum, acerbum, nigrum, grossum, sed bene digestum, desecatum, aureum, odoriferum et vetustum ${ }^{14}$.

${ }^{9}$ F. Testa, Capitula Regni Siciliae, I, cap. XCVIII, p. 93.

${ }^{10}$ N. Speciale, Historia sicula, p. 506; F. Testa, De vita, et rebus gestis Federici II, p. 221.

${ }^{11}$ A. Martellotti, I ricettari di Federico II, pp. 120-121.

${ }^{12}$ Ambivalente la considerazione in cui si tiene la frutta: si ritiene che vada in putrefazione all'interno dello stomaco, producendo terribili vapori nel corpo, P. Freedman, Il gusto delle spezie, p. 68; al contempo, per le sue qualità nutritive, è alimento consigliato ai malati e si pensa aiuti la digestione, M. Montanari, Vegetazione e alimentazione, I, p. 309.

${ }^{13}$ M. Ausécache, Des aliments et des médicaments, p. 257; M. Montanari, L'alimentazione, pp. 373-375. Vino de vernacia richiedeva Giacomo II nel 1306 al mercante genovese Cristiano Spinola de consilio phisicorum nostrorum ad nostri sanitatem corporis conservanda, J.E. Martínez Ferrando, Jaime II de Aragón, II, doc. 41.

${ }^{14} \mathrm{~K}$. Sudhoff, Ein diätetischer Brief, p. 5. Su Teodoro, fisico e speziale addetto alla preparazione di sciroppi e medicine per l'imperatore e la sua corte, $c f$. A. De Stefano, La cultura alla corte di Federico II, p. 42; Historia Diplomatica, V, 2, pp. 750-751. 
Se la cucina è terreno ideale dello scontro, ma anche dell'incontro e dello scambio fra culture diverse ${ }^{15}$, la Sicilia sveva della prima metà del XIII secolo si rivelava, a corte per lo meno, amalgama originale di sapori di provenienza diversa, in un orizzonte culturale in cui dietetica e gastronomia procedono in simbiosi, senza conflitti tra l'idea di piacere e quella di salute ${ }^{16}$, e in cui gli autori dei testi medici stabiliscono una connessione tra cucina e medicina ${ }^{17}$ des sortes de sæeurs siamoises, separatesi con il progresso della civiltà $^{18}$. Simbiosi incarnata perfettamente da Federico II, imperatore tra cucina e dietetica: salutista per quanto riguarda il cibo, sceglieva di seguire uno stretto regime alimentare; la sua corte, anche attraverso il suo impulso, era terreno fertile per la codificazione di un ambizioso trattato di scienza culinaria scritto in latino per meglio inserirsi nella manualistica scientifica ${ }^{19}$ che mischiava ricette arabe e tradizioni europee, all'insegna di una scoppiettante miscela di sapori. Il manuale, Liber de coquina che riprende altri ricettari in volgare ed è ispirato alla trattatistica medica ${ }^{20}$, comprendeva in una versione in toscano pervenutaci una sezione conclusiva di cibi per malati: piselli freschi, riso, mandorle, ceci, cavoli, triglie ${ }^{21}$.

Abitudini alimentari che diventano gusti, applicazione di principi dietetici integrati nello schema mentale di chi confeziona i cibi e di chi mangia $^{22}$, contribuivano alla diffusione di una cucina che deve soddisfare i palati e fungere da terapia.

${ }^{15}$ M. Montanari, Cucina povera, cucina ricca, p. 105.

${ }^{16}$ M. Montanari, Il cibo come cultura, p. 69.

${ }^{17}$ M. Nicoud, Savoirs et pratiques, p. 240.

${ }^{18}$ D. Jacquart, La nourriture et le corps, p. 260 e ibidem, p. 261 sulla corrispondenza tra quattro elementi e quattro umori.

${ }^{19}$ A. Martellotti, I ricettari di Federico II, pp. 117, 99, 134. Sull'aderenza dei ricettari alla cucina reale, G. Rebora, La cucina medievale, pp. 1497-1525.

${ }^{20}$ A. Martellotti, I ricettari di Federico II, pp. 5-19.

${ }^{21}$ M. Mulon, Deux traités inédits, pp. 396-420; F. Zambrini, Il libro della cucina, pp. 83-88. Successive attestazioni di area siciliana vedono la stessa mescolanza di testi di cucina e rimedi di carattere medico-pratico, P. Musso, Ricette di cucina siciliana, pp. 175-187.

${ }^{22}$ B. Laurioux, La cuisine des médecins, p. 143. Il gusto medievale è plasmato dalle credenze dietetiche, si digerisce meglio ciò che si mangia con piacere, e i sapori sono "fenomeno terapeutico di primaria importanza", J.-L. Flandrin, Condimenti, p. 387. Sul ruolo del gusto, C. Burnett, The Superiority of Taste, pp. 230-238. 


\section{CIBO PER LA CORTE}

Dele mele bullito co le noci, detto nucato. Togli mele bullito e schiumato, con le noci un poco peste e spezie cotte insieme: bagnati la palma de la mano coll'acqua et estendilo: lassa freddare e dà a mangiare. E puoi ponere mandole e avellane in luogo di noci. Libro della cucina del sec. XIV

Nel dodicesimo libro del Crestià, Eiximenis mentre parla di politica dà spazio all'alimentazione che si conviene al principe: il medico deve essere presente quando il sovrano mangia e beve, a controllare qualità e quantità, il maggiordomo tenga vicino i rimedi atti ad affrontare perills quis poden esdevenir en taula, l'avvelenamento ${ }^{23}$. Con competenze e professionalità diverse ma speculari medico e cuoco $^{24}$-probabilmente con una certa soggezione del secondo nei confronti del primo- hanno il delicato e difficile compito di tutelare la salute del $\mathrm{re}^{25}$ : a provacare la morte, a cinquantaquattro anni, di Martino il Vecchio re d'Aragona e Sicilia, sarebbe stato un cocktail di medicine e cibi afrodisiaci muy exquisitos, somministratigli per favorire il concepimento di un erede ${ }^{26}$.

Accanto al medico e fidati come il medico, un'équipe dalle competenze plurime si occupa, dalla natura alla messa in tavola, della salute del re, ad esempio l'esperto di erbe conosce, raccoglie, conserva e trasforma le piante medicinali. Nel 1407 il magister catanese Giovanni Monsono esaminato dal protomedico -specifica magistratura con ruolo di organo di controllo centrale su ogni tipo di attività in campo medico creata alla fine del XIV secolo da Martino d'Aragona ${ }^{27}$ - e risultato peritum et expertum circa herborum cognicionem, diventava l'herbularium di re Martino; aveva facoltà di raccogliere et secum ferre le erbe medicinali ubi voluerit, per prati, boschi, selve, giardini ${ }^{28}$.

${ }^{23}$ F. Eiximenis, Dotzè, II, 2, p. 161. Assicurare la presenza del medico o dei medici alla tavola del principe è compito del maggiordomo; questi si occupa inoltre delle vivande che devono essere "moltes e bones e bé endressades, segon l'estil de la nació dels convidats", ibidem, pp. 161-162. Sulle virtù del corno di unicorno contro i veleni, D. Santoro, La cura delle donne, pp. 785-786.

${ }^{24}$ Sulla definizione di "cuoco galenico", che basa le sue scelte sulla scienza dietetica (tecniche di cottura, modalità di accostamento fra le vivande, scelta dei vini, ordine del pasto), M. Montanari, Il cibo come cultura, p. 66.

${ }^{25}$ Compito del medico di corte diventa, anche, la ricerca di un equilibrio tra necessità mediche ed esigenze del gusto; più difficile da appurare la cultura medica del cuoco che deve comunque possedere una serie di conoscenze per le quali si appoggia al medico, B. Laurioux, Cuisine et médecine, p. 226.

${ }^{26}$ J. Zurita, Anales, 4, p. 930; M.R. Lo Forte Scirpo, C'era una volta una regina, p. 219.

${ }^{27}$ Sulla creazione dell'ufficio di protomedico, D. Santoro, La rete aperta, pp. 144-146.

${ }^{28}$ P. Sardina, Tra l'Etna e il mare, p. 215; ASP, Real Cancelleria, reg. 46, f. 292. Sul ruolo dei giardini nella medicina medievale, C. Opsomer-Halleux, The Medieval Garden, pp. 95 ss. 
Base della farmacologia popolare nel suo ambiguo carattere di scienza empirica e rituale magico ${ }^{29}$, erbe, spezie e piante compongono quell' arsenale terapeutico che provvede al riequilibrio dell'organismo, sulla scia della concezione ippocratica e galenica secondo cui la malattia ha origine da uno scompenso dei quattro umori ${ }^{30}$. Nel 1397 per loro usu necessariu i due Martino ordinano pepe (stimolante dell'appetito), cannella (digestiva), zafferano (calmante ed eupeptico), chiodi di garofano (dalle virtù anestetiche $\mathrm{e}$ antisettiche) ${ }^{31}$. Erbe e altri sapores (spezie, condimenti, salse) curano e correggono certi effetti alimentari ${ }^{32}$ e la farmacopea -basata sull'impiego di prodotti vegetali e animali combinati fra loro e talvolta con minerali- rientra a pieno titolo nell'ambito della cultura alimentare ${ }^{33}$. Arte culinaria e sapere medico finivano per attingere a un sapere collettivo, a un linguaggio comune che passa dai trattati di dietetica ai libri di cucina e viceversa ${ }^{34}$ e tra cibi che nutrono e cibi che curano, alimenti e medicamenti, la frontiera resta fluida e mobile ${ }^{35}$. Il medico si occupa di quello che si porta a tavola e compie incursioni in campo culinario $^{36}$, interessato alla manipolazione di cibi che assicurino la salute; il cuoco deve conoscere le proprietà mediche degli alimenti e, quando decide un tipo di cottura (arrostire secca un cibo mentre lo riscalda, bollire riscalda e aggiunge umidità... $)^{37}$, è consapevole delle conseguenza in senso gastronomico e fisico ${ }^{38}$.

A capo della cucina del signore, il cuoco deve possedere un vasto repertorio di piatti raffinati e originali -favorita dall'espansione della politica aragonese nell'isola, dal XIV secolo, si faceva strada l'influenza della cucina aragonese $-{ }^{39}$, non mettere a rischio la salute della famiglia e degli ospiti, garantire riserve di cibo ${ }^{40}$. Due onze erano la ricompensa che Martino il Gio-

\footnotetext{
${ }^{29}$ M. Montanari, Alimentazione, p. 209. Su erbe, rimedi e pratiche magico-religiose in uso nell'isola, D. Santoro, La rete aperta, pp. 149-152.

${ }^{30}$ M. Ausécache, Des aliments et des médicaments, p. 249.

${ }^{31}$ ASP, Real Cancelleria, reg. 29, f. 9r (6.9.1397).

${ }^{32}$ L. Moulin, La vita quotidiana, p. 77.

${ }^{33}$ M. Montanari, Alimentazione, p. 208.

${ }^{34}$ F. Pucci Donati, Dietetica, p. 130. Cf. B. Laurioux, Entre savoir et pratiques, pp. 59-71.

${ }^{35}$ B. Laurioux, Cuisine et médecine, p. 224.

${ }^{36}$ Arnau de Villanova e Maino de' Mainieri inserivano ad esempio ricette alimentari analoghe a quelle dei libri di cucina, P. Gil Sotres, Le regole della salute, p. 423.

${ }^{37}$ Sui modi di cottura, che implicano un rapporto diverso con l'aria e con l'acqua, C. LéviStrauss, Le triangle culinaire, pp. 9-19.

${ }^{38}$ Scegliere un metodo di cottura serve a correggere gli umori naturali di un cibo che può essere dannoso per chi lo consuma, T. Scully, L'arte della cucina, p. 52.

${ }^{39}$ B. Laurioux, Cucine medievali, p. 369.

${ }^{40}$ T. Scully, L'arte della cucina, p. 274. Sull'aspetto "ingannevole" della cucina medievale e la spettacolarizzazione connessa, J. Verdon, Il piacere, pp. 112 ss.
} 
vane, re di Sicilia, destinava nel 1399 al capocuoco di Casa reale, Guglielmo Feran, da dividere cum aliis sociis ${ }^{41}$.

Artigiano di estrazione popolare formatisi con l'apprendistato, il cuoco può conquistare un posto di rilievo nella considerazione del $\mathrm{re}^{42}$. Capocuoco alla corte di Giacomo II, Guillem Gallifa aveva seguito la famiglia reale in Sicilia: nel 1287 si trova a Palermo ${ }^{43}$, quattro anni dopo a Messina, pagato per il suo lavoro 8 onze d'oro ${ }^{44}$; con il suo signore che gli aveva assegnato case e altri beni ad Augusta, a pochi chilometri da Siracusa (beni che erano stati riassegnati e per i quali Giacomo nel 1294 da Barcellona scriveva al fratello Federico in Sicilia perché si occupasse della $\cos ^{45}$ ), faceva poi ritorno in Catalogna. Nel 1306 Guillem chiedeva al re di inviare a Barcellona un medico con il consiglio del quale avrebbe potuto provvedere ai pasti (les viandes e la manera de lur menjar) degli infanti. Il re da Valenza rispondeva di avere intenzione di inviare uno dei medici di corte ma invitava Guillem -mentre los infans estien sans-a non cercare il sostegno del medico per l'alimentazione dei figli e confidare piuttosto nella volontà di Dio: o, se proprio fosse stato necessario, chiedere consiglio ai medici mastro Bernat o mastro Nicolau ${ }^{46}$.

La realizzazione di pietanze spesso complicate e la presenza di un gran numero di persone che si occupano delle varie fasi, dal fuoco alla tavola, richiede che la cucina sia un laboratorio attrezzato di braccia e strumenti in grado di gestire bisogni e necessità molteplici.

La cucina del Palazzo arcivescovile di Palermo, desumiamo dall'inventario dei beni dell'arcivescovo Paolo Visconti ${ }^{47}$, appare dotata e ben arredata: la dispensa magna contiene cinque barrilia ad opus apportandi uvas, due tavole calabresi, varie botti piene di vino malvatico, rosso, moscatello; la dispensa parvula botti e carratelli vuoti e pieni, due quartane de terra. Nel magazzino, alquanto spoglio, si conservano dodici iarre vacue cathalaniske, un ligiu de libris, un barile cum tonnino intus fetente, altri due barili ad opus tonnini vuoti. Tra gli attrezzi da cucina utili a capire come il cuoco manipola, grattugia, dosa, condisce, affetta, frigge, sono presenti palette e padelle di fer-

${ }^{41}$ G. Beccaria, Spigolature, pp. 28, 153. Sul personale di cucina alla corte di Pietro III, J. Trenchs, Casa, corte y cancilleria de Pedro el Grande (1276-1285), pp. 75-79.

${ }^{42} \mathrm{G}$. Rebora, La cucina medievale, p. 1518. Su prestigio e potere dei medici di corte siciliani in età aragonese, D. Santoro, Medici del re, pp. 87-104.

${ }^{43}$ P. Burgarella, Le imbreviature, docc. 280, 392: Gallifa compare come testimone.

${ }^{44} \mathrm{G}$. La Mantia, Codice diplomatico dei re aragonesi, II, doc. 5.

${ }^{45}$ Ibidem, docc. 267, 297.

${ }^{46}$ J.E. Martínez Ferrando, Jaime II de Aragón, II, doc. 46; ACA, Cancillería, reg. 140, f. 7r.

${ }^{47}$ Appartenente all'Ordine carmelitano, Paolo Visconti, vescovo di Mazara, era stato eletto nella diocesi palermitana il 6 settembre 1469, C. Eubel, Hierarchia, II, p. 211. 
ro, una grattalora -di solito poco presente negli inventari siciliani, la grattugia per il formaggio è segnale di una cucina raffinata ${ }^{48}$, un mortaio di marmo cum pistone de ligno - $\mathrm{i}$ cibi vengono pestati, filtrati, macinati, triturati (per $\mathrm{i}$ piatti ritenuti dannosi il consiglio del medico era modificarli con l'aggiunta di alimenti dalle qualità opposte ${ }^{49}$ )-, due bilance (una romanisca, l'altra siciliana), sei taglieri di legno, un crivo ad opus ordei (setaccio si specifica per l'orzo), tre mensure ad opus mensurandi oleum: indizio questo, di una mensa ricca, dal momento che in genere i grassi usati in Sicilia come alimento erano animali, e l'olio, prezioso, veniva impiegato a crudo e per lo più dagli ecclesiastici (derrata tra l'altro di uso liturgico ${ }^{50}$ ), presente non a caso nella cucina del Palazzo arcivescovile. Il vescovo e la corte si nutrono di cibi conditi con olio, legumi soprattutto, preziosa fonte di proteine, all'insegna della varietà: tra le riserve del Palazzo ci sono ceste con fave, lenticchie e ceci, farro di Napoli e farro siciliano (la farina di farro si può usare per fare il pane), i saporiti ceci neri da utilizzare per zuppe o passati; e poi le immancabili mandorle da impiegare in preparazioni farmaceutiche, dolciarie o pestate come condimento per salse e triti; e ancora casse cum vermichelli e maccarruna. La mensa del vescovo comprende a proposito una coclearia ad opus de maccarruni ${ }^{51}$, il cucchiaio da maccheroni, portato a Palermo dagli artigiani del legno del Val Demone $^{52}$, segno che la pasta giocava una parte rilevante nell'alimentazione di corte, arcivescovile o regia: nel 1413 i vicegerenti si occupavano dell'estrazione, esente da tassazione, dal porto di Siracusa di otto cantari di vermicelli da inviare in Catalogna ${ }^{53}$.

Sostanza curativa per eccellenza, sconosciuto alla cucina classica greca e romana, lo zucchero è uno degli alimenti cardine della cucina e della farmacopea ${ }^{54}$, rimedio facilmente accessibile, largamente diffuso e

${ }^{48}$ H. Bresc, G. Bresc-Bautier, Cucina e tavola, p. 26.

${ }^{49} \mathrm{~T}$. Scully, L'arte della cucina, pp. 112-113.

${ }^{50}$ Sui grassi animali usati nell'alimentazione in Sicilia, ACFUP 2, pp. 158-159. Su diffusione e consumo dell'olio nell'alto medioevo, M. Montanari, L'alimentazione contadina, pp. 396-402. Sull'uso di burro e altri grassi animali, e sulla funzione dei condimenti, M. Montanari, Condimento, fondamento, pp. 27-51. Nell'isola come altrove l'olio serve soprattutto all'illuminazione, pesce e carne si conservano sotto sale, I. Peri, La Sicilia dopo il Vespro, p. 63. A conferma della preziosità del prodotto, un bando emanato a Palermo nel 1462 prevedeva l'obbligo, per quanti possedessero olio, di denunciarlo entro due giorni dall'emanazione, pena 10 onze e il sequestro del bene, ACP, Atti del Senato, cassetta 35/3, f. 7r.

${ }^{51}$ ASP, Notai defunti, Giacomo Randisi, reg. 1157, ff. 181v-188 (4.12.1476).

${ }^{52}$ G. Bresc-Bautier, Pour compléter, II, p. 436. Sulla diffusione progressiva di piatti, bicchieri, tovaglie, posate, R. Sarti, Vita di casa, pp. 174-178.

${ }^{53}$ ASP, Real Cancelleria, reg. 48, f. 61v.

${ }^{54}$ M. Barceló Crespí, A. Contreras Mas, Farmàcia i alimentació, p. 199. Assunto della medicina ippocratica e galenica è l'interrelazione farmaco-alimento, per cui quello che esiste in natura può diventare medicina ed essere assunto come alimento, ibidem. 
amato nelle corti per usi svariati: confetti di zucchero, polveri, empiastri, acque, fiori, erano tra le res medicinales fornite dall' aromatario Zatorra a Maria durante la sua infirmitas ${ }^{55}$. Non alimento in verità lo zucchero ma un temps, une catégorie du monde, sostanza che come il vino è praticamente un'istituzione ${ }^{56}$. La sua natura calda e umida, identica al temperamento umano, lo rende presenza universale nelle prescrizioni mediche ${ }^{57}$ (sciroppi, elettuari, julep: confezione farmaceutica con base di zucchero o miele) e in cucina: moderatamente caldo come il miele, esercita sui cibi un' azione correttiva meno potente di sale ${ }^{58}$, spezie, condimenti agri, acidi; il suo utilizzo è tale che tutta la cucina può intendersi come un'operazione dolcificante ${ }^{59}$.

L'accresciuta domanda di prodotti alimentari di lusso come confetti, marmellate, confetture, ricercati dalle corti di tutta Europa portava a un aumento della produzione. In particolare dopo il 1410, diventava straordinaria l'espansione dei confetti siciliani a base di mandorle, anice, coriandolo, e di dolci apprezzati come la cedrata, con buccia di cedro e la cucuzata, a base di zucca candita con zucchero, colorati e dolcissimi ingredienti della cassata siciliana. In occasione delle loro visite a Palermo, ambasciatori e viceré ricevono doni ghiotti: nel giugno 1417 ad Elionor, moglie del viceré Antoni Cardona, vengono offerte confetture di zucca e cedro, confetti di mandorle, anice e coriandolo, zucchero fine, costati alla città 7 onze e 5 grani $^{60}$.

La corte di Alfonso ne consuma grandi quantità. Nell'ottobre 1451 lo speziale palermitano Andrea de Lianora forniva una serie di prodotti per un costo complessivo di 6 onze, 24 tarì, 18 grani: si trattava di confezioni di zucchero infra amidolas, coliandrum et anasum e di sciroppo infra poma, pira, cucuzatam, scorchas citri et cothona, trasportate dalla bottega di Andrea ad litus maris, riposte in due barili e in una cassa contenente diciotto barattoli di vetro, burnie, piene di sciroppo, eviden-

\footnotetext{
${ }^{55}$ D. Santoro, Zucchero e acqua di rose, pp. 130-131. Sulle forniture di Zatorra e altri aromatari alla corte di Martino, ibidem, pp. 134-135.

${ }^{56}$ R. Barthes, Pour une psycho-sociologie, p. 978.

${ }^{57}$ T. Scully, L'arte della cucina, pp. 208-209.

${ }^{58}$ Sulle virtù del sale (essenzialmente la capacità essiccativa), celebrato nei ricettari antichi e medievali e nei trattati di dietetica, M. Montanari, Alimentazione, pp. 183-191. Sul vasto uso del sale nella farmacopea antica e medievale, ibidem, p. 188.

${ }^{59}$ J.-L. Flandrin, Condimenti, p. 388.

${ }^{60}$ ACP, Atti del Senato, cassetta 26, f. 14r; M. Ouerfelli, Le Sucre, p. 594. Elionor de Villena era la prima moglie di Cardona che nel 1421 sposa Margherita Peralta, Gran Enciclopèdia Catalana, 4, p. 408.
} 
temente per essere spedite ad Alfonso ${ }^{61}$ che si trovava in quel momento a Torre del Greco ${ }^{62}$.

Qualche tempo prima, negli anni confusi di Giovanna II, diviso il regno di Napoli tra Angiò e Aragona, Alfonso aveva dato mandato per una spedizione da Palermo a Napoli, con il buscaino di Giovanni de Sancto Iohanne (il costo era 372 onze, 24 tarì, 10 grani), caricato di tutto l'occorrente per andare in soccorso castrorum et insolarum Neapolis. Dettagliato l'elenco delle merci: tonnina, ceci, mandorle (il trasporto dal Cassaro di Palermo a la marina era costato 10 grani), cutugna, cucuzata, sporte di passuli e ficu, sei rotoli di coriandolo, anice e mandorle, quindici rotoli e mezzo di confetti di puma, pira, cutugna et cucuzata in xiruppu di mela; due scatole, marzapani, in cui erano stati riposti i confetti bianchi, 6 burnia in cui erano stati posti i confetti di sciroppo. In circostanze speciali come una spedizione, si rendevano necessari alimenti indispensabili, di consumo quotidiano: in questo caso l'approvvigionamento si limita ad alcuni prodotti di difficile alterazione, ceci, tonno, uva passa e fichi, mandorle ${ }^{63}$ e una serie di confetti. Segue l'elenco delle medicine inviate: notiamo la ripetizione di prodotti inseriti nell'elenco degli alimenti (uva passa, fichi secchi, zuccaru di misturi, anice, mandorle, pepe nero, cucuzata, coriandolo, usato in farmacia per le proprietà stomatiche, sudorifere e carminative) ${ }^{64}$ più una serie di prodotti specifici ad affrontare una missione armata: argento vivo, gomma arabica, trementina, salgemma, corallo bianco, bolo armenico, allume di rocca, incenso, cassia, verderame, cardamomo, mirra ${ }^{65}$.

\footnotetext{
${ }^{61}$ ASP, Real Cancelleria, reg. 86, ff. 88v-89. Burnia era il temine usato nell'isola per indicare gli albarelli e in generale i vasi degli speziali, V. Mortillaro, Nuovo dizionario, ad vocem. Su frutta, spezie, condimenti, dolci e confettura nella corte di Carlo III di Navarra, F. Serrano Larráyoz, La Mesa del Rey, pp. 211-222.

${ }^{62}$ A. Giménez Soler, Itinerario del rey don Alfonso, p. 270. Nel gennaio 1452, la spesa alla corte siciliana di Alfonso (che si trovava nel napoletano, ibidem, p. 271) per spezie, zucchero, vermicelli, era 141 onze, 4 tarì, 8 grani, ASP, Real Cancelleria, reg. 86, ff. 170v-171r.

${ }^{63}$ Le qualità nutrizionali di uva passa e fichi erano state messe in rilievo da Galeno che considerava invece poco nutrienti le mandorle, De alimentorum facultatibus, pp. 273-282 e p. 299.

${ }^{64}$ Sulle virtù medicinali di certi alimenti, M. Nicoud, Expérience de la maladie, p. 346. Sulle medicine dell'armata di Barcellona nel 1454, V. Mata Ventura, X. Sorní Esteva, Equipament sanitari, pp. 64-67.

${ }^{65}$ ASP, Conservatoria di registri, Debiti della corte, reg. 1062, ff. 390-397 (27.1.1426). Ringrazio il dottore Alessandro Silvestri della segnalazione del documento. Nel gennaio 1426 Alfonso si trova a Valenza, A. Giménez Soler, Itinerario del rey don Alfonso, p. 80. Sul vettovagliamento del viaggio a Nizza che Ferdinando I d'Aragona doveva realizzare nel 1415 per incontrare Benedetto XIII, E. Sarasa, La alimentacion de un rey aragonés, pp. 223-227.
} 


\section{CIBO PER LA CITTÀ}

"Pasta al burro, galletti abbracciati con guarnimento di patate, uova fritte, insalata, albicocche, nespole e domestici, e finalmente la cassata. Non c'è male, eh?' Leonardo Sciascia, Per un ritratto dello scrittore da giovane

La salute di un individuo dipende, prescrive Galeno, dalla sua alimentazione e si mantiene con un "regime" adatto e adattato, che tenga conto della sua complessione, attività, del posto occupato in società, del clima e della stagione ${ }^{66}$, dell' età e dunque, ad esempio, la nutrizione in età avanzata deve seguire regole precise. Emblematico che in un documento notarile stilato a Palermo alla fine del Quattrocento venga dato rilievo alla cura che i coniugi Bartolomeo de Castellis, notaio, e Isabella prestavano già da diversi anni nei confronti della madre di questa Ianna-in decrepita etate constituta est- ospitandola in eorum domo e accudendola, maxime in alimentand $o^{67}$. La nutrizione in età adulta, in base al regime galenico, prevede cibi digeribili, da mangiare più volte al giorno sempre in quantità limitata; proibiti pesce, legumi; consigliati uova, miele, latte di asina e capra, verdure calde e umide, frutta secca, pollo; il vino è raccomandato per le sue virtù ricostituenti e le qualità gustative; il pane consentito con una percentuale maggiore di crusca; la carne di vitello, agnello, porco, è ammessa solo se tenera $^{68}$.

Il consumo di carne -cibo del potens ${ }^{69}$ che marca la differenza tra ceti sociali (e pone, in teoria, problemi medici e religiosi), il secondo per importanza dopo il pane- aumenta nell'isola dopo la metà del Trecento e la carne è assegnata ai lavoratori come parte del salario ${ }^{70}$. Carne, pane e companaggio, forniscono calorie sufficienti ai settantasette saraceni che lavorano nel castello di Catania ${ }^{71}$.

${ }^{66}$ Y. Grappe, Sulle tracce, 160. Un regime ad personam, osservava Celso nel De medicina, non può rimanere rigido nel tempo ma va ritoccato almeno quattro volte l'anno, in corrispondenza delle stagioni, I. Mazzini, Alimentazione e medicina, p. 196. Nell'alto medioevo si era invece diffusa una letteratura dedicata alla dietetica che circola sotto forma di calendari: a seconda della stagione o del mese consigliato un certo tipo di regime alimentare, F. Pucci Donati, Dieta e calendari, pp. 209-219.

${ }^{67}$ ASP, Notai defunti, Giacomo Randisi, reg. 1157, ff. 290-291r (27.2.1477): in virtù dell "innatum amorem vigentem" tra lei e la figlia e dei "servicia" prestati da figlia e genero, Ianna donava a Isabella 15 onze "in iocalibus" a completare la dote.

${ }^{68} \mathrm{P}$. Gil Sotres, Le regole della salute, pp. 433-434.

${ }^{69}$ Sulla necessità che il potens mangi molta carne, M. Fiano, Il banchetto regio, 637-682.

${ }^{70}$ S.R. Epstein, Potere e mercati, p. 172.

${ }^{71}$ ASP, Real Cancelleria, reg. 47, f. 23r (8.11.1409). 
In una hiérarchie des goúts siciliana, la carne di maiale, pecora, capretto viene preferita nel XV secolo a quella di vitello ${ }^{72}$. Nel Macello Magno, alla Vucciria di Palermo, si vendono i caldumi, le interiora di animale, in questo caso montone macellato, caratteristico cibo da strada ${ }^{73}$ il cui gusto, pur essendo prodotto di scarto della macelleria, attraversa i gruppi sociali ${ }^{74}$. Il consumo isolano si attesta nel XV secolo a circa 20-22 kg pro capite annui, valore superiore a quello di altre regioni dell' area mediterranea ma più basso rispetto alla media europea ${ }^{75}$.

Vari i tipi di carne consumati a Palermo, come risulta dalle mete imposte nel 1411: silvestres (di daino, porco, colombo, conigli denudati seu absque pelle), salate (porcine, supprissate porcine, saucize porcine, carnes troyne), fresche (di vitello, maiale, vacca, agnello, pecora, castrato, carnes bovis et tauri, carnes troye $)^{76}$. Anni dopo un bando avrebbe proibito la vendita di agnelli maschi sia dentro che fuori città, pena una multa 20 onze e, ad arbitrio della corte, la prigione ${ }^{77}$. I danni provocati da sconfinamenti di buoi in proprietà vicine, danni consistenti se si tratta di campi di grano, si fanno frequenti nella documentazione palermitana della seconda metà del Quattrocento ${ }^{78}$, segno anche di un mutato paesaggio isolano per cui, aumentata la pressione demografica, si riducono gli spazi liberi per l'allevamento ${ }^{79}$.

Assieme al pane-personaggio invadente che riempie la storia d'Europa ${ }^{80}$ - ortaggi, frutta, piccola cacciagione sono i cibi della quotidianità, i cibi di tutti, venduti nelle botteghe per un consumo giornaliero ${ }^{81}$. Inserita in un'alimentazione quotidiana anche la pasta (vermicelli, maccheroni ${ }^{82}$ ) che come il

${ }^{72}$ M. Aymard, H. Bresc, Nourritures et consommation, p. 594.

${ }^{73}$ ASP, Notai defunti, Giacomo Randisi, reg. 1157, f. 88v (11.11.1476). Nel Libro della cucina del sec. XIV la ricetta per i caldumi prevede una cottura delle budella crude di vacca fritte nel lardo con cipolla e poi bollite con tuorlo d'uovo, mollica di pane, spezie, erbe, F. Zambrini, Il libro della cucina, p. 41.

${ }^{74}$ M. Montanari, Gusti del Medioevo, p. 190.

${ }^{75}$ S.R. Epstein, Potere e mercati, p. 172.

${ }^{76}$ ACP, Atti del Senato, cassetta 23, f. 2r. Su mete, prezzi e imposte sulle carne a Barcellona nel XV secolo, R. A. Banegas López, Comer carne, pp. 329-355. Nella città catalana il concetto di "salud alimentaria" si evolve dopo la Peste del 1348: "pasa de ser una responsabilidad personal del consumidor a ser una responsabilidad colectiva asumida por los dirigentes municipales", R. A. Banegas López, El consumo de carne, p. 93.

${ }^{77}$ ACP, Atti del Senato, cassetta 35/3, f. 5r (18.10.1462).

${ }^{78}$ ACP, Atti del Senato, cassetta 35/3, f. 7r (5.11.1462) e f. 43r (15.9.1462).

${ }^{79}$ A. Giuffrida, Considerazioni, p. 590.

${ }^{80}$ F. Braudel, Le strutture, p. 117.

${ }^{81}$ ASP, Notai defunti, Giacomo Randisi, reg. 1157, f. 2v (4.9.1476): stipulata una società di quattro anni per una bottega a Palermo "ad vendendum panem, fructus, olera, ortalicia, candelas, fructus uvarum, aves, candelas sepi et alias res".

${ }^{82}$ ASP, Real Cancelleria, reg. 98, f. 39 (17.7.1455). 
pane, dall'impasto alla cottura si fa in casa: in un contratto notarile stipulato a Palermo nel 1337, Isabella si impegna a lavorare presso Benenato de Casanova ad facendum servicia domus eiusdem conductoris per un anno, con la specificazione che dovrà coquinare, impastare, abluere pannos e lactare la figlia di quello de die et de nocte ${ }^{83}$.

Grande protagonista è ovviamente il frumento, oru et argentu del Regno $^{84}$. A ondate ripetute emerge una situazione critica: la penuria può essere dovuta ai flussi di approvvigionamento, condizionati da epidemie e carestie. Esigenti, i palermitani: dopo l'epidemia di peste che da Messina dilaga con forza a Catania ${ }^{85} \mathrm{e}$ in tutta l'isola, nel 1351 pretendono di acquistarne di prima qualità, bonum et comestibilem dal momento che, tengono ad evidenziare, non sumus sicut ceteri alii de nonnullis Sicilie partibus frumentum non bonum edere nec habere ${ }^{86}$.

Nel settembre 1392 Martino viene informato che la città patitur caristia et maxime victualium a causa del divieto di acquistare viveri nei dintorni e scarseggia di carne et hoc propter distracionem animalium que distrahuntur per mare ${ }^{87}$. Nel novembre Martino e Maria, re e regina di Sicilia e Martino, duca di Montblanc rassicurano i palermitani: per rifornire la città di viveri $\mathrm{e}$ frumento viene vietata l'esportazione di vettovaglie e bestiame dai porti di Termini e Alcamo e dato ordine di mandare a Palermo plus ki quantu frumentu si atrova, così da non patire defectu di caristia nexuna ${ }^{88}$. Nel dicembre 1406 Palermo è una città ferita, al punto che quando si aspetta la nascita dell'erede al trono, considerandu li affanni di quissa universitati, lo stesso re ordina di evitare spese eccessive per i festeggiamenti ${ }^{89}$.

Anni difficili sono per l'isola, e soprattutto per Palermo, anche quelli successivi a Caspe, all'insegna di uno stato permanente di guerriglie e carenze: nel dicembre 1413 pretore e giurati scrivono ai vicegerenti facendo presente la penuria in cui versa la città, dovuta da una parte a una quantità di frumento promessa e non ancora consegnata, dall'altra al fatto che le navi non sono potute partire a causa del maltempo. Palermo rischia lo spopolamento,

\footnotetext{
${ }^{83}$ ASP, Notai defunti, Salerno de Pellegrino, reg. 4, f. 61 (6.10.1337): Isabella porterà con se la figlia Altadonna e oltre a vitto e alloggio avrà una tunica di lana, una "suctana", un "faciolum" di lino; nel caso si ammali dovrà ricevere assistenza.

${ }^{84}$ I. Peri, Restaurazione, pp. 37-45.

${ }^{85}$ Michele da Piazza, Cronaca, I, cap. 29: "glandule ille ad modum nucis crescebant, deinde ad modum ovi galline...".

${ }^{86}$ ACFUP 9, Introduzione, a cura di L. Sciascia, p. XXVII e doc. 37.

${ }^{87}$ ACFUP 10, doc. 192: la città chiede di proibire l'esportazione di animali da macello e il permesso di acquistare animali ovunque nell' isola per macellarli.

${ }^{88}$ ACFUP 10, docc. 235, 245. Cf. P. Sardina, Palermo, pp. 333-337.

${ }^{89}$ ACFUP 12, doc. 89.
} 
a causa di multi ki andirannu ad abitari undi poczanu atruvari vita ${ }^{90}$. Nel febbraio 1414 veniva imposto il divieto di panificare con altro frumento che non fosse quello della corte e di vendere in piazza il pane cui non fosse stato apposto il sigillo della corte ${ }^{91}$. Altra situazione critica più tardi, nel 1462: un bando di settembre obbliga quanti possiedano frumento fuori città, a portarlo in città: entro ottobre se il frumento si trova nel raggio di 30 miglia, entro novembre oltre 30 miglia, stabilendo per i trasgressori la pena di morte e la confisca dei beni ${ }^{92}$. A novembre si faceva esplicita proibizione di avvicinarsi di notte ai depositi di frumento alla Marina, pena la forca ${ }^{93}$. Inoltre, il carico di grano in uscita dalla città su muli e cavalli avrebbe dovuto seguire un percorso stabilito, attraverso le porte Termini e Mazara ${ }^{94}$.

Di grande interesse, perché ci informa sulle modalità di conservazione e distribuzione, è un capitolo di ordinanze del 1414 dell'universitas di Sciacca: nessuno avrebbe dovuto, pena una multa di 50 onze, mutari lu furmetu di una fossa ad altra infussandu et sfussandu; dal momento in cui il frumento veniva messo nelle fosse, lì doveva essere lasciato a meno di situazioni estreme, ad esempio che la fossa si riempisse d'acqua. Era importante infatti che il frumento, ammassato in fosse scavate nel terreno per essere utilizzato successivamente, si mantenesse al chiuso, al riparo dall'umidità, evitando la penetrazione di aria e parassiti. Lo stesso documento entra poi nel merito dei metodi di controllo, vigilanza, smistamento: i vicegerenti davano ordine che in ogni luogo in cui ci fosse stato exitu di furmentu, presidiassero le porte della città e delle terre persone deputate a scrivere e annotare la quantità di frumento, sia le quantità ki si mictinu intra la terra quantu killi ki restanu di fora fossati oy sfossati, tantu per serviri di lu serenissimu signuri re et di soy diricti quantu per utilitati et comoditati di la universitati e delle singole persone, onde evitare inconvenenti ki potissiru suchediri non sapendusi la certa quantitati di li furmenti ${ }^{95}$.

Dopo l'epidemia di peste a metà Trecento, la coltivazione delle cannamele decollava nei dintorni di Palermo: i sopravvissuti e le generazioni successive avrebbero trovato nello zucchero un dolcificante apprezzabile più del

\footnotetext{
${ }^{90}$ ACP, Atti del Senato, cassetta 25, ff. 6v-7r.

${ }^{91}$ ACP, Atti del Senato, cassetta 25, f. 52v.

${ }^{92}$ ACP, Atti del Senato, cassetta 35/3, f. 1v. Sugli Acta Curie Felicis urbis Panormi, fonte cittadina conservata presso l'Archivio Storico del Comune di Palermo, D. Santoro, Acta Curie Felicis Urbis Panormi (1311-1410), pp. 235-244.

${ }^{93}$ ACP, Atti del Senato, cassetta 35/3, f. 6v.

${ }^{94}$ ACP, Atti del Senato, cassetta 35/3, f. 4v.

${ }^{95}$ ASP, Real Cancelleria, reg. 49, f. 159.
} 
miele e un sussidio all'alimentazione tonico e ricostituente ${ }^{96} . \mathrm{Al}$ contempo spezia e articolo da farmacia, lo zucchero inizialmente è venduto nelle spezierie come energetico, sotto forma di confetti e confetture ${ }^{97}$, anche da molti speziali siciliani proprietari di trappeti ${ }^{98}$.

Ampio pure il consumo e il commercio di miele: nel luglio 1417 capitano, pretore e giudici di Palermo stabiliscono che gli straguluni, specie di carri da trasporto, possano accedere in città per il trasporto del miele, del vino ma non di altro, pena un'ammenda di un'onza" ${ }^{99}$. Ingrediente principale nella vasta gamma di dolci siciliani, terapeutico con le sue proprietà solutive, emollienti, cicatrizzanti ${ }^{100}$, il miele è impiegato per confezionare una serie di rimedi adottati dalla medicina popolare siciliana ${ }^{101}$.

Il primo trentennio del XV secolo è caratterizzato da un'espansione straordinaria dell'industria zuccheriera siciliana, avanzata al punto che specialisti palermitani sono inviati a Barcellona per l'impianto di stabilimenti di lavorazione in Catalogna ${ }^{102}$. La moltiplicazione dei trappeti dentro Palermo creava una serie di problemi, contribuiva ad esempio a deteriorare, a causa del transito eccessivo di carri, l'aspetto della città; nel maggio 1417 si procede a regolamentare (Ordinacio facta per dominos vicereges super transitum carrociarum) la circolazione dei carri che trasportano cannamele e legna nei trentuno trappeti intra urbem, prevedendo per i carri differenti e dettagliati percorsi e il pagamento annuale di 6 tarì da utilizzare in reparacione et restauracione viarum civitatis ${ }^{103}$.

Come per la carne, tra il 1370 e il 1440 il prezzo del formaggio a Palermo diminuisce in maniera consistente, e crescono i consumi ${ }^{104}$. Nel 1411 venivano imposte le mete per i tipi più consumati: formaggio di pecora e di

${ }^{96}$ I. Peri, Restaurazione, p. 51. Sull'impatto della peste nell'isola, L. Sciascia, Malattia e salute, pp. 41-48.

${ }^{97}$ Cf. l'elenco degli alimenti e delle medicine a base di zucchero venduti nelle botteghe di quattro speziali maiorchini tra la fine del XV secolo e l'inizio del XVI, M. Barceló Crespí, A. Contreras Mas, Farmàcia i alimentacio, pp. 209-211 e 213-216.

${ }^{98}$ Cf. D. Santoro, Profili di speziali, pp. 65-73. Su speziali cristiani ed ebrei nell'isola, J.-P. Bénézet, Pharmacie et médicament, pp. 185-192.

${ }^{99}$ ACP, Atti del Senato, cassetta 26, f. 3v.

${ }^{100}$ Cf. I. Naso, Apicoltura, cera e miele, pp. 228-229, 234-235.

${ }^{101}$ G. Pitré, Medicina popolare siciliana, pp. 382-383.

${ }^{102}$ S.R. Epstein, Potere e mercati, p. 206. Sulla "precocità" siciliana, la rapida espansione dell'attività e la crisi alla fine del XV secolo, M. Ouerfelli, Le Sucre, pp. 149-179. Nel 1305 Giacomo chiede di avere in Catalogna due schiavi siciliani "sarracenos", esperti nella lavorazione del cotone e della canna da zucchero, J.E. Martínez Ferrando, Jaime II de Aragón, II, doc. 33.

${ }^{103}$ ACP, Atti del Senato, cassetta 26 , ff. 12v-13; M. Ouerfelli, Le Sucre, pp. 702-704.

${ }^{104}$ S.R. Epstein, Potere e mercati, p. 172. 
vacca (4 grani a rotolo), caciocavallo, apprezzato e diffuso ${ }^{105}$ (5 grani a rotolo), tuma (16 denari a rotolo), ricotta (14 denari a rotolo $)^{106}$. A regolarne l'esportazione la città nel 1412 vieta, senza licenza apposita, l'estrazione per mare o per terra di cascavalli sagimii et burru oy altra graxa, pena la confisca di beni ${ }^{107}$.

Il ripetersi di simili provvedimenti è rivelatore della volontà delle autorità cittadine di controllare la fornitura di generi -vino, grasso, carne, pesce- considerati di prima necessità imponendo, anche in situazioni di non emergenza, misure a carattere preventivo (ad esempio il divieto di acquistare derrate per rivenderle) in modo da assicurare agli abitanti una certa autosufficienza alimentare ${ }^{108}$. Nel 1407 a Palermo, per calmierare i prezzi ed evitare speculazioni, venivano imposte le mete di grano ( 7 tarì a salma), orzo (6 tarì $\mathrm{e}$ 10 grani), carne (ordine per i macellai di vendere la carne vaccina un denaro a rotolo, pena un'onza di multa), latticini (10 denari a rotolo la ricotta) ${ }^{109}$, pesce: con l'obbligo per i pescatori di venderlo soltanto in pescheria e, grande o piccolo che fosse, a peso e non a resta ${ }^{110}$. La vigilanza delle città era rivolta anche al rispetto, da parte dei venditori, di pesi e misure: nel maggio 1462 pretore e giurati di Palermo attraverso un bando obbligavano tucti quilli persuni ki usanu et teninu tummina mundelli et menczi mundelli per actactari et vindiri di recarsi entro dodici giorni dal tumminaru della città, l'aggiustatore di pesi e misure che abitava a Ballarò, per il controllo e la taratura degli strumenti di misurazione; stabilivano inoltre che i controllo avessero cadenza mensile ${ }^{111}$.

Prodotto garantito dalla geografia del luogo, il pesce. I tipi di pesce consumati nel Quattrocento sono abbastanza vari e -in un panorama alimen-

\footnotetext{
${ }^{105}$ A metà Quattrocento il palermitano Masocta de Castillucio vende a due concittadini quaranta cantari di caciocavallo buono e stagionato, prodotto "in salmoria bulluta ut dicitur vulgariter", proveniente da una sua mandria di vacche "azzaccanata usque ad lac finitum", ASP, Notai Defunti, Notaio Antonino Candela, reg. 575, f. 153: il caciocavallo era venduto a 10 tarì d'oro a cantaro. Nel 1405 Nicola Raya di Palermo vende a Suffeni Misiria cinque cantari di formaggio "crapini boni, novi, sani, sucidi et assupti", ASP, Notai defunti, Francesco Riccio, reg. 451, f. 27. Sul formaggio kosher siciliano, S. Simonsohn, The Jews in Sicily, 1, doc. 208 (9.10.1214), 218 (1.6.1241). Sull'identità alimentare ebraica, H. Bresc, Arabi per lingua, pp. 69-73.

${ }^{106}$ ACP, Atti del Senato, cassetta 23, f. 2r.

${ }^{107}$ ACP, Atti del Senato, cassetta 23, f. 6v. Sull'uso dei grassi animali nella cucina europea (che non dipende solo dalla disponibilità e dai bisogni legati al clima ma dalle tendenza del gusto), J.-L. Flandrin, Le goût et la nécessité, pp. 369-391.

${ }^{108}$ Per l'area pedemontana $c f$. A.M. Nada Patrone, Il cibo del ricco, pp. 24-26.

${ }^{109}$ ACFUP 12, docc. 108, 122, 132.

${ }^{110}$ ACFUP 12, docc. 125, 127. Veniva ripreso in questo caso un "capitulum" emanato da Federico III nel 1330 che vietava ai pescatori di vendere pesce a peso o "a resta" in altri luoghi (a casa, a mare, nelle barche) che non fossero la pescheria, $c f$. M. De Vio, Felicis et fidelissimae urbis, pp. 109, 117-118; ACFUP 2, pp. 155-156; P. Sardina, Palermo, pp. 376-377.

${ }^{111}$ ACP, Atti del Senato, cassetta 35/3, f. 14r.
} 
tare in cui i medici raccomandano le specie più care, mentre il consumo maggiore è il pesce secco e salato ${ }^{112}$ - freschi: pisces grossi et murrini (nel 1412 venduti 4 tarì a rotolo); sarde, asinelli (naselli) e vopi (boghe), venduti 3 grani a rotolo; pesci piccoli di sciabica (pescati a strascico con la rete, venduti 2 grani e mezzo a rotolo) ${ }^{113}$, pesci bianchi (nel 1414 venduti 5 grani a rotolo), murene, salpe, occhiate e aguglie ( 3 grani a rotolo $)^{114}$, tonno, acquistato spesso dai monasteri, ad esempio nel 1414 da quelli benedettini di S. Maria de Novaluce e di S. Nicolò L'Arena, nel catanese ${ }^{115}$ e venduto anche nelle botteghe degli speziali1 ${ }^{116}$. Il pezzo più pregiato è il tarantello ${ }^{117}$, tipo di filetto meno grasso della ventresca ma ugualmente saporito: nel 1416 venivano imposte mete supra rebus venalibus tra cui pane di crusca e tarantellu di mari ${ }^{118}$.

Il vino infine, il più utilizzato tra i liquidi nella preparazione di medicamenti, largamente diffuso e consumato nell'isola sia bianco che nero: bastano delle circostanze negative per alterare la media dei consumi, come dimostrano anonime vicende quotidiane utili a definire e fissare il quadro economico e sociale. È uno scenario drammatico quello che emerge a tratti dalla supplica al viceré, in un volgare pregnante, di Geronimo Amato che nel 1450 aveva acquistato la gabella del vino di Patti: quartuchu unu per quartara di la universitati di la dicta chitati, per 10 onze. Quell' anno si era verificata una congiuntura sfavorevole: la penuria di frumento aveva fatto aumentare i costi a 2 tarì et plui lu thuminu, et maxime lu pani di Plaza ki valia 10 tarì e 2 grani vel circa, mentre ai poviri plui li era necessariu acactari pani per ipsi et li figli per campari ki acactari vinu. Le rendite previste non si erano realizzate a causa anche di una recrudescenza di peste: per accaxuni di la infeccioni si erano visti pochi forestieri e non era nemmeno stata realizzata la fiera cittadina che costituiva l'introito maggiore della gabella; sino a quel momento i tabernari che solitamente non aveva problemi di spaccio, non avevano venduto vino e quillu ki vindinu est achitu. Geronimo -che sottolineava di avere servitu bene l'universitas, ambasciatore a la maiestati di lu signuri re (a Catanzaro ventotto giorni, a Palermo per altri fachendi cinquantadue giorni) - si trovava

${ }^{112}$ P. Gil Sotres, Le regole della salute, p. 422.

${ }^{113}$ ACP, Atti del Senato, cassetta 23, f. 5r (19.2.1412).

114 ACP, Atti del Senato, cassetta 25, f. 52r. Sugli attrezzi, H. Bresc, Il vocabolario della pesca, pp. 11-23.

${ }^{115}$ ACP, Atti del Senato, cassetta 25, f. 27r; f. 28r.

${ }^{116}$ ACP, Atti del Senato, cassetta 26, f. 2v: ingiunzione del 1417 a cinque speziali cittadini di non vendere per tutto l'anno in corso candele o tonnina nelle loro botteghe, pena un'ammenda di 3 onze da versare alla curia.

${ }^{117}$ G. Caracausi, Dizionario onomastico, II, ad vocem. Sulle varie parti del tonno e il corrispettivo tariffario, P. Sardina, Palermo, pp. 374-375.

${ }^{118}$ ACP, Atti del Senato, cassetta 26, f. $2 \mathrm{v}$. 
in difficoltà, aveva carricu di octo figli, specificava di essere povero e di aver perduto tempo e denaro ( 2 onze) per ricogliri ipsa cabella. Il suo reclamo cadeva a vuoto: per multu ki eu protestassi non fui ascoltatu, vessato dai giurati che pretendevano di avere, cosa non consentita dal re, due salme di frumento a testa e lo avevano preso di punta, ki si lu putissiru fari muriri non si sarebbero tirati indietro. Per rifarsi del mancato pagamento della colletta, 4 tarì, gli avevano sequestrato un cavallo che valeva 2 onze, ki sindi campava ipsu et li figli, e già erano quarantaquattro giorni che lo tenevano a lu fundacu taliter ki est quasi mortu. Geronimo ribadiva che non avrebbe pagato la colletta prevista e chiedeva di avere indietro il cavallo ${ }^{119}$.

\section{RICREAZIONE}

descriveva a Lodovico il monastero dei Benedettini come un luogo di eterna delizia, dove la vita passava, senza cure dell'oggi e senza paure del domani, tra lauti conviti, sontuose cerimonie, gaie conversazioni e scampagnate gioconde.

E quando finalmente Lodovico entrò novizio a San Nicola poté riconoscere che la madre aveva detto la verità, perché il corno dell'abbondanza pareva rovesciarsi continuamente sul monastero e la vita vi scorreva facile e lieta. Federico De Roberto, I Viceré

Chiamato a ricoprire la carica di giurato di Polizzi, Matteo Salamone si ammalava, suchidenduli la pilagra et lu mali di lu yancu; nel tentativo di guarire da quell'infermità era andato, cum sua casa et famigla, a Termini cum animu di tornari arreri ma lì rimaneva più del previsto, sino a lu presenti; trascorrevano, dall'estate in cui si era ammalato, tre anni ${ }^{120}$. A pochi chilometri da Palermo, Termini Imerese era conosciuta per le sue acque termali utilizzate anche nel caso di malattie della pelle quali appunto la pellagra, causata dall'insufficienza di vitamina B. Tra i mezzi igienici e terapeutici per conservare la salute, $\mathrm{i}$ bagni ${ }^{121}$ erano stati presi in considerazione da Galeno che ne aveva calcolato funzione e modalità a seconda della temperatura dell'acqua.

\footnotetext{
${ }^{119}$ ASP, TRP, Lettere viceregie e Dispacci, reg. 1, ff. 74v-75r (22.8.1450).

${ }^{120}$ ASP, TRP, Num. provv., reg. 51, f. 41r (30.4.1459). Della segnalazione archivistica si ringrazia il dottore Francesco Barna. Sulle principali carenze, vitaminiche e proteiche, nell'alimentazione medievale, M. Aymard, Pour l'histoire de l'alimentation, pp. 439-442. Su dieta, malattie e carenze alimentari nella Sicilia medievale, H. Bresc, Prima dello scavo antropologico, pp. 337-352. Sulla gerarchia sociale dei consumi, M. Aymard-H. Bresc, Nourritures et consommation, pp. 597-598.

${ }^{121}$ Regimen sanitatis ad regem Aragonum, 3.2-7; $f f$. P. Gil Sotres, Regimen sanitatis, pp. 629-643.
} 
Le proprietà curative delle acque termali sono utilizzate per curare malattie reumatiche, respiratorie, dermatologiche, sfruttando siti presenti nell'isola ${ }^{122}$ : Lipari e Segesta sono ricordati da Plinio il quale consiglia, dopo il bagno nelle sorgenti calde, di frizionare il corpo con olio ${ }^{123}$.

Acque medicinali aromatizzate con fiori d'arancio, rosa, melissa, sono rimedio per la cura di varie affezioni: nel 1399, quando dominus rex passus fuit illum modicum accidens, l'aromatario di corte aveva confezionato per Martino elettuari, acque medicinali, acqua di rose, oli, per un costo complessivo di 19 onze, 9 tarì, 10 grani ${ }^{124}$. Due anni prima il re aveva speso 15 onze per acqua nanfa, acqua profumata ottenuta dalla distillazione di fiori d'arancio, di rose, di viole, cum quedam conca ${ }^{125}$. Le acque di rosa, gelsomino, arancio, conservate in conche, in fiaschi di ferro, bronzo, cristallo venivano spruzzate, come nella novella di Boccaccio, dopo i bagni caldi e seguite, a mo' di conforto, da confetti e vino ${ }^{126}$.

Tra le spese di corte di Martino, ancora, 12 tarì servivano nel 1398 all'acquisto di zibetto e muschio per la regina Maria ${ }^{127}$ : si trattava di due essenze profumate di origine animale rare e preziose, difficili da procurare, usate anche come rimedio (il muschio come sedativo e antispasmodico) conservate in piccole fiale di vetro e vasetti d'argento e d'oro ${ }^{128}$.

Influenzati dalla medicina galenica, i regimina sanitatis avevano dato spazio all'azione esercitata sull'uomo da tutto quello che lo circonda ${ }^{129}$. La salute nasce un equilibrio tra la complessione individuale e il mondo esterno, sono pertanto indispensabili le res non naturales, aria e ambiente, esercizio e riposo, sonno e veglia, alimenti e bevande, ciò che si ingerisce ed espelle, le emozioni: tra quelle positive la gioia che, dal punto di vista igienico, non conosce controindicazioni. I medici suggeriscono per suscitarla non solo una corretta alimentazione ma di godere della vita da tutti i punti di vista: nutrirsi adeguatamente, trascorrere del tempo con gli amici, vivere in accordo al proprio rango sociale, ascoltare buona musica. Passioni che si accompagnano a

${ }^{122}$ R. Gregorio, Dell'uso in Sicilia dei pubblici bagni, p. 738.

${ }^{123}$ Plinio, Storia Naturale, IV, XXXI.61.

${ }^{124}$ D. Santoro, Zucchero e acqua di rose, p. 140.

${ }^{125}$ ASP, Miscellanea Archivistica, II, reg. 35, f. 131r.

${ }^{126}$ Sui bagni in Sicilia, compreso quello a Palermo descritto da Boccaccio nel Decameron (che testimonia la fedeltà a tecniche e abitudini degli hammam arabi), L. Sciascia, Il bagno di madama Biancofiore, pp. 152-166.

${ }^{127}$ ASP, Miscellanea Archivistica, II, reg. 34, f. 69r; G. Beccaria, Spigolature, p. 122.

${ }^{128}$ P. Lanza di Scalea, Donne e gioielli, p. 94. Su acque, oli, profumi, D. Santoro, Zucchero e acqua di rose, pp. 140-145.

${ }^{129} C f$. M. Nicoud, Les régimes de santé, pp. 339-395. Aria e cibo i due aspetti più importanti per la salute: l'aria con la respirazione, gli alimenti con la digestione penetrano direttamente all'interno dell'organismo senza mediazione alcuna, M. Nicoud, Savoirs et pratiques, p. 243. 
un raffreddamento del cuore e del corpo, da evitare per i loro effetti negativi sulla salute, sono invece ansia, paura, collera, tristezza ${ }^{130}$.

Dunque svago, intrattenimento, risate: tra i familiares e fideles di Maria e Martino -appassionato di musica, impara a suonare l'arpa- ci sono due instriones, Bernardo Çarovira e Nicola Drago ${ }^{131}$. E banchetti, con tutto il loro contorno.

La tavola di corte è associata spesso alla festa, a uno scenario fatto di suoni, colori, stoviglie, stoffe: oro, pietre preziose, abiti sontuosi e un abbondante rinfresco venivano esibiti in occasione delle nozze di Federico IV con la principessa napoletana Antonia del Balzo celebrate a Messina nel 1374, nel Palazzo regio riparato e addobbato; veniva acquistata della tela pro pilastris factis tempore nupciarum nostrarum, per decorare qualche padiglione costruito appositamente ${ }^{132}$. E avevano coinvolto tutta la città nei festeggiamenti le nozze, nel 1352 ad Agrigento, del conte messinese Enrico Rosso con la figlia di Federico Chiaromonte: suoni, canti e un banchetto sfarzoso con vasellame d'oro e d'argento all'interno del palazzo di famiglia; pane e vino distribuiti per tre giorni all'esterno ${ }^{133}$. Il cibo di re e signori, il cibo dei ricchi va condiviso. Martino re di Sicilia che, nel Natale 1398, aveva distribuito a tutta la famiglia reale vesti e panni nuovi ${ }^{134}$, l'anno seguente aveva festeggiato a $\mathrm{Ca}$ tania nascita e battesimo del figlio Pietro. Martino e Maria avevano offerto, dopo il rito religioso per celebrare il quale era stato necessario aptare organa et fontem della cappella di Palazzo, un grande pranzo al quale invitavano i nobili più vicini alla famiglia reale: il banchetto era allietato da musiche eseguite da pifferai calabresi, e confetti di zucchero venivano serviti in tre eleganti brocche d'argento ${ }^{135}$.

Per il riposo del re e della corte vengono creati luoghi di piacere per il corpo e per lo spirito, spazi "privilegiati" che, veicolati da tradizioni arabe, avevano caratterizzato la Sicilia normanna costellata da loca amoena, parchi e giardini il cui idillico miraggio di primavera eterna era legato all'acqua ${ }^{136}$. Spazi preclusi ai più -la création de ces lieux de loisir est un acte de pouvoir

${ }^{130}$ P. Gil Sotres, Le regole della salute, pp. 403-404, 428-429.

${ }^{131}$ G. Beccaria, Spigolature, pp. 21-23, 122; ASP, Real Cancelleria, reg. 18, f. 12 (2.5.1393).

${ }^{132}$ G. Cosentino, Le nozze, pp. 38-39; ASP, Real Cancelleria, reg. 13, f. 6v. Su tessuti e vesti, e su arredi e corredi nelle case siciliane, H. Bresc, Une maison, pp. 119-123. Sul corredo di Eleonora di Sicilia, regina d'Aragona, terza moglie di Pietro il Cerimonioso, e della corte reale a Barcellona, L. Sciascia, Scene e costumi, pp. 32-44.

${ }^{133}$ S. Fodale, Tra gioco e rito, p. 12; I. Peri, Restaurazione, p. 172.

${ }^{134}$ G. Beccaria, Spigolature, pp. 26, 137.

${ }^{135}$ I. Peri, Restaurazione, pp. 172-173; G. Beccaria, Spigolature, pp. 57-58, 159-162, 169.

${ }^{136}$ S. Tramontana, Il Regno di Sicilia, p. 381. 
royal $^{137}$ - che trovano altri mezzi per contrastare malattie ed epidemie, e affrontano la paura del contagio all'insegna di un senso pratico per cui l'aria, sulla scia della medicina galenica, è ritenuta tra le principali responsabili della salute dell'uomo: un bando del 1422 stabilisce di non consentire l'ingresso a Palermo a chi venga da Messina undi fussi la influencia di la pistilenza, e pena una multa di 4 onze, di non di ospitare qualcuno proveniente da quella zona o di non denunciarne la presenza ${ }^{138}$.

\section{CONCLUSIONI}

Mangiare e curarsi. Due dimensioni fortemente connesse al corpo, ai suoi bisogni, piaceri, necessità. Due dimensioni dense di sfumature sociali e locali, religiose ed economiche, che implicano conoscenze e pratiche diverse a seconda dell'età e del sesso ma soprattutto del ruolo sociale. Due dimensioni che con gli abiti, la moda, la casa non sono solo "cose" ma "linguaggi" 139 e, in quanto tali, implicano una serie di sovrastrutture. Niente controlli, paletti, regole per i sovrani il cui rapporto con il cibo è all'insegna della quantità massiccia, dell'abbondanza. Quando, nel 1300, Giacomo II ordina ai giurati di Sella di preparare la cena per l'arrivo suo e di Bianca d'Angiò, l'elenco comprende, tra l'altro, galline, vino, pane, quattro libbre di pepe, zafferano, sessanta montoni, tre vacche, due vitelli da latte, due maiali carnium salarum $^{140}$ : le "orge di carne" sono elemento peculiare della "tavola dei ricchi"141 e solo successivamente si avvia un percorso per cui la raffinatezza più che la quantità diventava segno distintivo ${ }^{142}$.

Che si tratti delle spezie caricate dal porto di Palermo e spedite a Napoli per le necessità di Alfonso o del carico di formaggi (doentes peces de formages redons) che nell'agosto 1397 arrivava da Maiorca al palazzo di Barcellona su ordine di Martino il Vecchio ${ }^{143}$, la tavola a corte viene imbandita con i cibi graditi al sovrano o qui li sien sanes e profitoses, segons lo temps e segons la condició e complexió e estament de sanitat del príncep, a consell de bons metges ${ }^{144}$.

\footnotetext{
${ }^{137}$ J. Blanchard, Le corps du roi, p. 200.

${ }^{138}$ ACP, Atti del Senato, cassetta 29, f. 6.

${ }^{139}$ F. Braudel, Le strutture, p. 301.

${ }^{140}$ J.E. Martínez Ferrando, Jaime II de Aragón, II, doc. 5.

${ }^{141}$ F. Braudel, Le strutture, p. 168.

${ }^{142}$ Cf. M. Montanari, La fame e l'abbondanza, pp. 71-76.

${ }^{143}$ D. Girona Llagostera, Itinerari del rey en Martì, (40) 18.

${ }^{144}$ Cf. F. Eiximenis, Dotzè, II, 1, pp. 136-155.
} 
Fuori dalla corte l'alimentazione è fortemente connessa all'ambiente in cui si vive, all'organizzazione realizzata dal territorio per lo smistamento dei prodotti, alla reperibilità degli alimenti ${ }^{145}$. I provvedimenti annonari messi in atto dalle città consentono diversi piani di lettura e coinvolgono sfere molteplici ma testimoniano innanzi tutto il legame con flussi economici e specifiche situazioni per cui le scelte alimentari, specie nel corso del Quattrocento sono condizionate più che da umori e temperamenti ${ }^{146}$ da spazio e tempo, da denaro, condizioni climatiche, ritmi di approvvigionamento delle singole realtà urbane. Si mangia quello che si trova, quello che si può -il mercato locale appare in grado, tutto sommato, di garantire un'offerta di prodotti abbastanza ampia- e ci si ammala, come i re, di malattie legate a squilibri o carenze nutrizionali, calcoli alla vescica per esempio, per estrarre i quali nel 1397 un chirurgo di Nicosia veniva abilitato a esercitare per il Regno ${ }^{147}$. Abbienti e indigenti affrontano la malattia in modo diverso $^{148}$ : è con la chirurgia e non con rimedi sofisticati -le polveri lassative a base di prugne fornite nel 1398 da un aromatario palermitano a Martino re di Sicilia $^{149}$ - che i più possono curarsi. Per i chirurghi abituati a trattare ferite e infezioni di tipologia diversa, il protomedico del Regno di Sicilia Ingrassia stabiliva più tardi un tariffario preciso: 2 onze erano nel 1564 il compenso previsto per cauterizzare un'ernia, estrarre calcoli dalla vescica, guarire scrofole e cataratta ${ }^{150}$.

Mentre gli isolani gestiscono e subiscono un mercato che risente di congiunture non prevedibili e la loro dieta gratta su un numero vario ma pur sempre ristretto di alimenti e medicamenti, itineranti come le loro corti i sovrani hanno la possibilità di attingere da pozzi diversi. Il lusso si manifesta più che nei palazzi -che spesso in occasione di nozze, feste, ricevimenti, occorre riparare e adattare- nelle cose a diretto contatto con il corpo, i cibi ricercati e abbondanti, i bagni, gli abiti, i rimedi costosi, i luoghi di evasione a cui è legato il benessere della persona. Mangiare e prendersi cura di sé, insieme a tutto l'esibizionistico, assillante allestimento che ruota attorno a queste due dimensioni, permangono e si impongono come elementi che, se non garantiscono la salute, assicurano un più piacevole vivere.

${ }^{145}$ Sulle varianti locali dei consumi alimentari, G. Piccinni, Note, pp. 610-611.

${ }^{146}$ Una cultura che riguarda i comportamenti relativi a mangiare e bere diventa allora patrimonio di un pubblico che, senza ricorrere al medico, è libero di selezionare, rielaborare, adattare, $c f$. M. Nicoud, Savoirs et pratiques, pp. 242, 247.

${ }^{147}$ Il chirurgo veniva abilitato ad esercitare la parte della chirurgia riguardante "curam et manualem operationem rupturarum sive erniarum, apostematum testiculorum et extraccionem lapidis de vessica", ASP, R. Canc., reg. 26, f. 125r.

${ }^{148}$ Cf. M.S. Mazzi, Salute e società, pp. 10-26.

${ }^{149} \mathrm{D}$. Santoro, Zucchero e acqua di rose, p. 135. Grande l'attenzione che medico e farmacista dovevano riservare alle medicine lassative: la scelta del tipo di rimedio era legata all'umore da evacuare, ibidem, pp. 137-138.

${ }^{150}$ G.F. Ingrassia, Constitutiones, p. 65. 


\section{BIBLIOGRAFIA CITATA}

Acta Curie Felicis Urbis Panormi, 2, Fisco e società nella Sicilia aragonese. Le pandette delle gabelle regie del XIV secolo, a cura di Rosa Maria Dentici Buccellato, Palermo, Municipio di Palermo, 1983.

Acta Curie Felicis Urbis Panormi, 9, Registro di lettere (1350-1351), a cura di Cecilia Bilello, Francesco Bonanno, Anna Massa, Introduzione di Laura Sciascia, Palermo, Municipio di Palermo, 1999.

Acta Curie Felicis Urbis Panormi, 10, Registro di lettere (1391-1393) e ingiunzioni (1324), a cura di Daniela Santoro, Palermo, Municipio di Palermo, 2002.

Acta Curie Felicis Urbis Panormi, 12, Registri di lettere atti bandi e ingiunzioni (1400-1401 e 1406-1408), a cura di Patrizia Sardina, Palermo, Municipio di Palermo, 1996.

Alessandro di Telese, De rebus gestis, in G. Del Re, Cronisti e scrittori sincroni napoletani, vol. I, Napoli, Dalla stamperia dell'Iride, 1845.

Ausécache, Mireille, Des aliments et des médicaments, "Cahiers de recherches médiévales et humanistes" 13 spécial (2006), pp. 249-258.

Aymard, Maurice; Bresc, Henri, Nourritures et consommation en Sicile entre XIVe et XVIIIe siècle, "Annales. Économies, Sociétés, Civilisations" 2-3 (1975), pp. 592-599.

Aymard, Maurice, Pour l'histoire de l'alimentation: quelques remarques de méthode, "Annales. Économies, Sociétés, Civilisations" 2-3 (1975), pp. 431-444.

Banegas López, Ramón Agustín, El consumo de carne y las mentalidades en el mundo urbano de la Baja Edad Media: Barcelona durante los siglos XIV y XV, "Ex Novo: Revista d'Història i Humanitats" 5 (2008), pp. 81-96.

Banegas López, Ramon Agustí, Comer carne y pagar impuestos: el impacto de las imposiciones municipales en el comercio barcelonés de carne durante el siglo XV, "Anuario de Estudios Medievales" 39/1 (2009), pp. 329-355.

Barceló Crespí, Maria; Contreras Mas, Antoni, Farmàcia i alimentacio: L'exemple del sucre a la Mallorca baixmedieval, "Bolletí de la Societat Arqueològica Lul-liana: Revista d'estudis històrics" 50 (1994), pp. 199-218.

Barthes, Roland, Pour une psycho-sociologie de l'alimentation contemporaine, "Annales. Économies, Sociétés, Civilisations" 16/5 (1961), pp. 977-986.

Beccaria, Giuseppe, Spigolature sulla vita privata di re Martino in Sicilia, Palermo, Tip. Lit. Salvatore Bizzarrilli, r.a. Messina, Intilla, 1993. 
Bénézet, Jean-Pierre, Pharmacie et médicament en Méditerranée occidentale (XIIIe-XVIe siècles), Paris, Honoré Champion, 1999.

Blanchard, Joël, Le corps du roi: mélancolie et "recreation". Implications médicales et culturelles du loisir des princes à la fin du Moyen Âge, in idem (ed.), Représentation, pouvoir et royauté à la fin du Moyen Âge, Paris, Picard, 1995, pp. 199-214.

Braudel, Fernand, Le strutture del quotidiano, Torino, Giulio Einaudi editore, 1982.

Bresc, Henri, Il vocabolario della pesca nella Sicilia del '300 e del '400, "Bollettino dell'Atlante linguistico mediterraneo" 16-17 (1974), pp. 11-23.

Bresc, Henri; Bresc-Bautier, Geneviève, Cucina e tavola a Palermo nel Tre e Quattrocento, in Atti IX Convegno Internazionale della Ceramica, Albisola, Centro ligure per la storia della ceramica, 1976, pp. 21-36.

Bresc, Henri, Prima dello scavo antropologico: cibo e salute dalle fonti archivistiche, "Archeologia medievale" 8 (1981), pp. 337-352.

Bresc, Henri, Une maison de mots: inventaires palermitains en langue sicilienne (1430-1456), "Bollettino del Centro di studi filologici e linguistici siciliani” 18 (1995), pp. 109-187.

Bresc, Henri, Arabi per lingua, Ebrei per religione, Messina, Mesogea, 2001. Bresc-Bautier, Genevieve, Pour compléter les données de l'archéologie: le role du bois dans la maison sicilienne (1350-1450), "Atti del Colloquio Internazionale di Archeologia Medievale”, Palermo-Erice, 20-22 settembre 1974, vol. II, Palermo, Istituto di Storia medievale, Università di Palermo, 1976, pp. 435-464.

Burgarella, Pietro, Le imbreviature del notaio Adamo de Citella a Palermo ( $1^{\circ}$ Registro: 1286-1287), Roma, Il Centro di ricerca, 1981.

Burnett, Charles, The Superiority of Taste, "Journal of the Warburg and Courtauld Institutes" 54 (1991), pp. 230-238.

Caracausi, Girolamo, Dizionario onomastico della Sicilia: repertorio storicoetimologico di nomi di famiglia e di luogo, vol. II, Palermo, l'Epos, 1994.

Cosentino, Giuseppe, Le nozze del re Federico III con la principessa Antonia del Balzo, Palermo, Alberto Reber, Libreria Carlo Clausen, 1895.

De Stefano, Antonino, La cultura alla corte di Federico II imperatore, Bologna, N. Zanichelli, 1950.

De Vio, Michael, Felicis et fidelissimae urbis Panormitanae selecta aliquot privilegia, Panormi, in Palatio senatorio per Dominicum Cortese, 1706.

Eiximenis, Francesc, Dotzè llibre del Crestià, II, 1-2, Girona, Col-legi Universitari de Girona - Diputació de Girona, 1986-1987. 
Epstein, Stephan R., Potere e mercati in Sicilia. Secoli XIII-XVI, Torino, Giulio Einaudi editore, 1996.

Eubel, Conradus, Hierarchia catholica medii aevi ab anno 1198 usque ad annum 1431 perducta, vol. II, Monasterii, Sumptibus et typis librariae Regensbergianae, 1914.

Fiano, Maria, Il banchetto regio nelle fonti altomedievali. Tra scrittura ed interpretazione, "Mélanges de l'École française de Rome - Moyen Âge" 115 (2003), pp. 637-682.

Flandrin, Jean-Louis, Condimenti, cucina e dietetica tra XIV e XVI secolo, in Flandrin, Jean-Louis; Montanari, Massimo, Storia dell'alimentazione, Roma - Bari, Laterza, 1997, pp. 381-395.

Flandrin, Jean-Louis, Le goût et la nécessité: sur l'usage des graisses dans les cuisines d'Europe occidentale (XIVe-XVe siècle), “Annales. Économies, Sociétés, Civilisations" 38/2 (1983), pp. 369-401.

Fodale, Salvatore, Tra gioco e rito: aspetti dell'identità urbana (il caso palermitano e siciliano), in El món urbà a la Corona d'Aragó del 1137 als decrets de Nova Planta (Actas XVII Congreso de Historia de la Corona de Aragón), vol. I, Barcelona, Universitat de Barcelona, 2003, pp. 9-18.

Freedman, Paul, Il gusto delle spezie nel Medioevo, Bologna, il Mulino, 2009.

Galenus, Claudius, De alimentorum facultatibus, edidit Georgius Helmreich, Leipzig - Berlin, Teubner, 1923. (Corpus medicorum graecorum; 4,2).

Gil Sotres, Pedro, Le regole della salute, in Storia del pensiero medico occidentale. 1. Antichità e Medioevo, a cura di Mirko Gremk, Roma - Bari, Laterza, 1993, pp. 399-438.

Gil Sotres, Pedro, Regimen sanitatis ad regem aragonum: Introducción, en García Ballester, Luis; McVaugh, Michael R. (eds.), Regimen sanitatis ad regem aragonum, (Arnaldi de Vilanova Opera Medica Omnia; X.1), Barcelona, Universitat de Barcelona - Fundació Noguera, 1996, pp. 471-904.

Giménez Soler, Andrés, Itinerario del rey don Alfonso de Aragón y de Nápoles, Zaragoza, Mariano Escar, 1909.

Girona Llagostera, Daniel, Itinerari del rey en Martì de Aragò (1396-1410), Barcelona, Anuari de l'Institut d'Estudis Catalans, 1916.

Giuffrida, Antonino, Considerazioni sul consumo della carne a Palermo nei secoli XIV e XV, "Mélanges de l'École française de Rome - Moyen Âge" 87/2 (1975), pp. 583-595.

Gran Encilopèdia Catalana, vol. IV. Barcelona, Enciclopèdia Catalana, 1973.

Grappe, Yann, Sulle tracce del gusto. Storia e cultura del vino nel Medioevo, Roma - Bari, Laterza, 2006. 
Gregorio, Rosario, Dell'uso in Sicilia dei pubblici bagni, in Opere rare edite ed inedite riguardanti la Sicilia, Palermo, Stab. tip. di Pietro Pensante, 1873, r.a. Sala Bolognese, Arnaldo Forni, 1977.

Grieco, Allen J. Alimentazione e classi sociali nel tardo Medioevo e nel Rinascimento in Italia, in Flandrin, Jean-Louis; Montanari, Massimo, Storia dell'alimentazione, Roma - Bari, Laterza,1997, pp. 370-380.

Historia Diplomatica, a cura di J.L.A. Bréholles, Parisiis, 1859, r.a. Bottega D'Erasmo, Torino, 1963.

I documenti inediti dell'epoca normanna in Sicilia, a cura di Carlo Alberto Garufi, Palermo, Società Siciliana per la Storia Patria, 1899.

Ingrassia, Giovanni Filippo, Constitutiones et Capitula, necnon et iurisditiones regii protomedicatus officii, cum pandectis eiusdem, reformatae ac in pluribus renovatae atque elucidatae à Ioanne Philippo Ingrassia huius Siciliae Regni, insularumque coadiacentium regio protomedico anno suae possessionis primo, Panormi, apud Ioannem Mattheum Maydam, 1564.

Jacquart, Danielle, La nourriture et le corps au Moyen Âge, "Cahiers de recherches médiévales et humanistes" 13 spécial (2006) pp. 259-266.

La Mantia, Giuseppe, Codice diplomatico dei re aragonesi di Sicilia, II, a cura di Antonino De Stefano-Francesco Giunta, Palermo, Società Siciliana per la Storia Patria, 1956.

Lanza di Scalea, Pietro, Donne e gioielli in Sicilia nel Medio Evo e nel Rinascimento, Palermo, C. Clausen, 1892, r.a. Bologna, Forni editore, 1971.

Laurioux, Bruno, Entre savoir et pratiques: le livre de cuisine à la fin du Moyen Age, "Médiévales" 14 (1988), pp. 59-71.

Laurioux, Bruno, La cuisine des médecins à la fin du Moyen Age, in Maladie, Médecines et Sociétés. Approches historiues pur le Présent, t. II, Paris, L'Harmattan et Histoire au présent, 1993, pp. 136-148.

Laurioux, Bruno, Cucine medievali (secoli XIV e XV), in Flandrin, Jean-Louis; Montanari, Massimo, Storia dell'alimentazione, Roma - Bari, Laterza,1997, pp. 356-370.

Laurioux, Bruno, Cuisine et médecine au Moyen Âge. Alliées ou ennemies?, "Cahiers de recherches médiévales et humanistes" 13 spécial (2006), pp. 223-238.

Lévi-Strauss, Claude, Le triangle culinaire, "Food \& History" 2/1 (2004), pp. 9-19.

Lo Forte Scirpo, Maria Rita, C'era una volta una regina... Due donne per un regno: Maria d'Aragona e Bianca di Navarra, Napoli, Liguori Editore, 2003.

Martellotti, Anna, I ricettari di Federico II: dal "Meridionale" al "Liber de coquina", Firenze, Leo S. Olschki, 2005. 
Martínez Ferrando, J. Ernesto, Jaime II de Aragón. Su vida familiar, vol. II, Barcelona, Consejo Superior de Investigaciones Científicas, 1948.

Martínez Ferrando, J. Ernesto, Jaume II, in Martínez Ferrando, Ernest.; Sobrequés, Santiago; Bagué, Enric, Els descendents de Pere el Gran. Alfons el Franc, Jaume II. Alfons el Benigne, Barcelona, Vicens Vives, 1961.

Mata Ventura, Víctor; Sorní Esteva, Xavier, Equipament sanitari de l'armada de la ciutat de Barcelona contra el corso capitanejada por Jaume Bertran (1454), "Drassana Revista del Museu Marítim" 11 (2003), pp. 62-75.

Mazzi, Maria Serena, Salute e società nel Medioevo, Firenze, La Nuova Italia Editrice, 1978.

Mazzini, Innocenzo, Alimentazione e medicina nel mondo antico, in Flandrin, Jean-Louis; Montanari, Massimo, Storia dell'alimentazione, Roma Bari, Laterza,1997, pp. 191-200.

Michele da Piazza, Cronaca (1336-1361), ed. Antonino Giuffrida, Palermo, ila palma, 1980.

Montanari, Massimo, Condimento, fondamento. Le materie grasse nella tradizione alimentare europea, in Cavaciocchi, Simonetta, Alimentazione e nutrizione secc. XIII-XVIII, Atti della ventottesima Settimana di studi, 22-27 aprile 1996, Istituto Internazionale di Storia Economica “F. Datini”, Prato, Firenze, Le Monnier, 1997, pp. 27-51.

Montanari, Massimo, L'alimentazione contadina nell'alto Medioevo, Napoli, Liguori, 1979.

Montanari, Massimo, Vegetazione e alimentazione, in L'ambiente vegetale nell'Alto Medioevo (Settimane di studio del Centro italiano di studi sull'alto Medioevo, XXXVII), 2 voll., Spoleto, presso la Sede del Centro, 1990, pp. 175-290.

Montanari, Massimo, La fame e l'abbondanza. Storia dell'alimentazione in Europa, Roma - Bari, Laterza, 1994.

Montanari, Massimo, Cucina povera, cucina ricca, "Quaderni medievali" 52 (2001), pp. 95-105.

Montanari, Massimo, La cucina scritta come fonte per lo studio della cucina orale, "Food \& History" 1/1 (2003), pp. 251-259.

Montanari, Massimo, Il cibo come cultura, Roma - Bari, Laterza, 2005.

Montanari, Massimo, Gusti del Medioevo: i prodotti, la cucina, la tavola, Roma - Bari, Laterza, 2012.

Mortillaro, Vincenzo, Nuovo dizionario siciliano-italiano, Palermo, Stamperia di Pietro Pensante, 1853.

Moulin, Léo, La vita quotidiana dei monaci nel Medio Evo, Milano, Mondadori, 1993. 
Mulon, Marianne, Deux traités inédits d'art culinaire médiéval, "Bulletin Philologique et Historique" 1 (1968), pp. 369-435.

Musso, Pasquale, Ricette di cucina siciliana in una farmacopea del Cinquecento, in Castiglione, Marina; Rizzo, Giuliano (eds.), Parole da gustare. Consuetudini alimentari e saperi linguistici, Palermo, Centro di studi filologici e linguistici siciliani - Dipartimento di scienze filologiche e linguistiche, 2007, pp. 175-187.

Nada Patrone, Anna Maria, Il cibo del ricco e quello del povero. Contributo alla storia qualitativa dell'alimentazione. L'area pedemontana negli ultimi secolo del Medio Evo, Torino, Centro Studi Piemontesi, 1981.

Naso, Irma, Apicoltura, cera e miele, in Uomo e ambiente nel Mezzogiorno normanno-svevo, Bari, Edizioni Dedalo, 1989, pp. 203-240.

Nicoud, Marilyn, Expérience de la maladie et échange épistolaire: les derniers moments de Bianca Maria Visconti (mai-octobre 1468), "Mélanges de l'Ecole française de Rome. Moyen-Age, Temps modernes" 112/1 (2000), pp. 311-458.

Nicoud, Marilyn, Savoirs et pratiques diététiques au Moyen Âge, "Cahiers de recherches médiévales et humanistes" 13 spécial (2006), pp. 239247.

Nicoud, Marilyn, Les régimes de santé au Moyen Âge. Naissance et diffusione d'une écriture médicale (XIIIe-XVe siècle), 2 voll., Rome, École française de Rome, 2007.

Opsomer-Halleux, Carmélia, The Medieval Garden and Its Role in Medicine, in Medieval Gardens, Washington, Dumbarton Oaks - Trustees for Harvard University, 1986, pp. 93-113.

Ouerfelli, Mohamed, Le Sucre: production, commercialisatione et usages dans la Méditerranée médiévale, Leiden - Boston, Brill, 2008.

Peri, Illuminato, La Sicilia dopo il Vespro. Uomini, città e campagne 12821376, Roma - Bari, Laterza, 1982.

Peri, Illuminato, Restaurazione e pacifico stato in Sicilia 1377-1501, Roma Bari, Laterza, 1988.

Piccinni, Gabriella, Note sull'alimentazione medievale, "Studi Storici: rivista trimestrale dell'Istituto Gramsci “ 23/3 (1982), pp. 603-615.

Pitrè, Giuseppe, Medici, chirurgi, barbieri e speziali antichi in Sicilia, secoli XIII-XVIII, Palermo, Reprint, 1992.

Pitrè, Giuseppe, Medicina popolare siciliana, S. Giovanni La Punta, Clio, 1993.

Plinio Secondo, Gaio, Storia Naturale, IV, Medicina e farmacologia, Libri 28-32, Torino, Giulio Einaudi editore, 1986.

Pucci Donati, Francesca, Dieta e calendari nell'Occidente latino altomedievale, "Food \& History" 2/2 (2004), pp. 209-219. 
Pucci Donati, Francesca, Dietetica e cucina nel Regimen Sanitatis di Maino de' Maineri, "Food \& History" 4/1 (2006) pp. 107-131.

Rebora, Giovanni, La cucina medievale italiana tra oriente e occidente, "Miscellanea storica ligure" 19 (1987), pp. 1431-1579.

Regimen sanitatis ad regem Aragonum, ed. L. García-Ballester e M. R. McVaugh, (Arnaldi de Villanova. Opera medica omnia; X.1), Barcelona, Publicacions de la Universitat de Barcelona - Fundació Noguera, 1996.

Regimen sanitatis: flos medicinae Scholae Salerni, ed. Andrea Sinno, Milano, U. Mursia, 1987.

Santoro, Daniela, Zucchero e acqua di rose: tra fiori, erbe e acque medicinali in Sicilia, alla corte di re Martino, "Schede medievali" 41 (2003), pp. 129-148.

Santoro, Daniela, Profili di speziali siciliani tra XIV e XVI secolo, "Mediterranea. Ricerche storiche" 9 (2007), pp. 63-76.

Santoro, Daniela, La cura delle donne. Ruoli e pratiche femminili tra XIV e XVII secolo, in Pacifico, Marcello; Russo, Maria Antonietta; Santoro, Daniela et al. (eds.), Memoria, storia e identità. Scritti per Laura Sciascia, Palermo, Mediterranea. Ricerche storiche 2011, pp. 777 801. (Quaderni Mediterranea. Ricerche storiche; 17).

Santoro, Daniela, La rete aperta. Pratica medica nel tardomedioevo siciliano, "Mediterranean Chronicle" 1 (2011), pp. 143-152.

Santoro, Daniela, Medici del re nella Sicilia aragonese, in Elisa, Andretta; Nicoud, Marilyn (eds.) Etre médecin à la cour (Italie, France et Espagne, XIII-XVIII siècles), Florence, Sismel, 2013, pp. 87-104. (Micrologus' Library; 52).

Sarasa, Esteban, La alimentación de un rey aragonés y su séquito a comienzos del siglo XV, in Manger et boire au Moyen Âge. Actes du Colloque de Nice 1(5-17 octobre 1982), t 2. Cuisine, manières de table, régimes alimentaires, Paris, Les Belles Lettres, 1984, pp. 223-231.

Sardina, Patrizia, Tra l'Etna e il mare. Vita cittadina e mondo rurale a Catania dal Vespro ai Martini (1282/1410), Messina, Sicania, 1995.

Sardina, Patrizia, Palermo e $i$ Chiaromonte splendore e tramonto di una signoria. Potere nobiliare, ceti dirigenti e società tra XIV e XV secolo, Caltanissetta - Roma, Salvatore Sciascia editore, 2003.

Sarti, Raffaella, Vita di casa. Abitare, mangiare, vestire nell'Europa moderna, Roma - Bari, Laterza, 1999.

Schmitt, Jean-Claude, Il gesto nel Medioevo, Roma - Bari, Laterza, 1990.

Sciascia, Laura, Il bagno di madama Iancofiore: l'eros come frontiera, "Quaderni medievali" 52 (2001), pp. 152-167. 
Sciascia, Laura, Malattia e salute a Palermo nel XIV secolo attorno alla peste nera, in Leone, Alfonso; Sangermano, Gerardo (eds.), Le epidemie nei secoli XIV- XVII, , Salerno, Laveglia Editore, 2006, pp. 33-48.

Sciascia, Laura, Scene e costumi: regalità e moda alla corte di Barcellona, in Colesanti, Gemma Teresa, Le usate leggiadrie. I cortei, le cerimonie, le feste e il costume nel Mediterraneo tra il XV e XVI secolo, Montella (AV), Centro Francescano di Studi sul Mediterraneo, 2010, pp. 32-44.

Scully, Terence, L'arte della cucina nel Medioevo, Casale Monferrato, Piemme, 1997.

Serrano Larráyoz, Fernando, La Mesa del Rey. Cocina y réginem alimentario en la corte de Carlos III el Noble de Navarra (1411-1425), Pamplona, Gobierno de Navarra, Departamento de Educación y Cultura, 2002.

Simonsohn, Shlomo, The Jews in Sicily, 1, Leiden - Koln, Brill, 1997.

Nicolò Speciale, Historia sicula, in Rosario Gregorio, Bibliotheca scriptorum qui res in Sicilia gestas sub Aragonum imperio retulere, I, Panormi, Ex Regio Typographeo, 1791.

Sudhoff, Karl, Ein diätetischer Brief an Kaiser Friedrich II. von seinem Hofphilosophen Magister Theodorus, "Archiv für Geschichte der Medizin", 9 (1915), pp. 2-9.

Testa, Franciscus, Capitula Regni Siciliae, I, Panormi, Excudebat Angelus Felicella, 1741.

Testa, Franciscus, De vita, et rebus gestis Federici II, Siciliae regis, Panormi, Excussit cum privilegio Cajetanus M. Bentivenga sub signo Ss. Apostolorum, propre plateam Bononiorum, 1775.

Storia dell'alimentazione, a cura di Jean-Louis Flandrin e Massimo Montanari, Roma-Bari, Laterza, 1997.

Tramontana, Salvatore, L'effimero nella Sicilia normanna, Palermo, Sellerio, 1988.

Tramontana, Salvatore, Giochi, feste, spettacoli, in Uomo e ambiente nel Mezzogiorno normanno-svevo, Bari, Edizioni Dedalo, 1989, pp. 319-342.

Tramontana, Salvatore, Il Regno di Sicilia. Uomo e natura dall'XI al XIII secolo, Torino, Einaudi, 1999.

Tramontana, Salvatore, Modelli alimentari di Sicilia, "Quaderni medievali" 52 (2001), pp. 6-17.

Trenchs, Josep, Casa, corte y cancilleria de Pedro el Grande (1276-1285), Roma, Bulzoni Editore, 1991.

Verdon, Jean, Il piacere nel Medioevo, Milano, Baldini \& Castoldi, 1999. 
Zambrini, Francesco, Il libro della cucina del sec. XIV, Bologna, Gaetano Romagnoli, 1863, r.a. Bologna, Libreria Editrice Forni, 1968.

Zurita, Jeronimo, Anales de la Corona de Aragón, edición preparada por Angel Canellas Lopez, vol. 4, Zaragoza, Institución "Fernando el Católico" (CSIC), 1978.

Fecha de recepción del artículo: noviembre 2012

Fecha de aceptación y versión final: mayo 2013 